\title{
Effects of Extremely Low Frequency Magnetic Field (ELF-MF) on Formalin Induced Chronic Pain in Mice
}

\author{
Mahsa Hadipour Jahromy*, Hossein Jafari, Sara Rezaee, Ahmad Jamshidi Mohajer \\ Herbal Pharmacology Research Centre, Faculty of Medicine, Tehran Medical Sciences Branch, Islamic \\ Azad University, Tehran, Iran \\ Email: "jahromymh@yahoo.com
}

Received 7 March 2016; accepted 19 April 2016; published 22 April 2016

Copyright (C) 2016 by authors and Scientific Research Publishing Inc.

This work is licensed under the Creative Commons Attribution International License (CC BY).

http://creativecommons.org/licenses/by/4.0/

(c) (i) Open Access

\begin{abstract}
Background: Effects of Extremely Low Frequency Magnetic Field (ELF-MF) in the elevation of blood sugar, cholesterol, triglyceride and reduction of withdrawal syndrome of morphine have been reported so far. Since pain is one of the main concerns in medicine and usually analgesic drugs are not much beneficial or cause considerable side effects, the present project was carried out with the aim of evaluating the effects of (ELF-MF) in chronic pain using formalin test in mice. Materials $\&$ Methods: In this experimental study, thirty two adult male mice were used and divided into 4 groups $(n=8)$. Three groups of animals exposed daily for thirty minutes to $25,50,75 \mathrm{HZ}$ (intense $250 \mu \mathrm{T}$ ) of electromagnet field for one week, respectively and one group considered as control with no exposure. At the end of a week, formalin test was performed. Responses to formalin were observed for sixty minutes. The results were compared between tests and control group. Findings: In acute phase of formalin test, ELF-MF reduced significantly the pain scores following formalin injection at all 3 frequencies, especially at 25 and $50 \mathrm{HZ}(\mathrm{p}<0.001)$ rather $75 \mathrm{HZ}(\mathrm{p}<0.05)$. In chronic phase of formalin test, ELF-MF reduced pain scores at 25 and $50 \mathrm{HZ}(\mathrm{p}<0.05)$. However, the most effective responses were obtained at $50 \mathrm{HZ}$ frequency. Conclusion: The findings show that ELF-MF is effective to reduce formalin induced chronic pain in mice at both acute and chronic phases.
\end{abstract}

\section{Keywords}

ELF-MF, Formalin-Induced Pain, Mice

\footnotetext{
${ }^{*}$ Corresponding author.
} 


\section{Introduction}

Electromagnetic spectrum encompasses a wide range of wavelengths and frequency. In fact, its scope is so vast that a logarithmic scale is necessary to review them [1]. We live in a world of energies and waves every day and we hear in the news and research content from risks such as mobile phones, televisions, radio, computer, mobile waves that are the most widely used. Many studies have shown that electromagnetic fields at low frequency have adverse effects on mental and physical health of living organisms [2] [3]. However, in some cases, aspects of treatment have been reported. The changes of cellular and molecular radiation waves depend on the duration of radiation exposure, the permeability of the tissue and heat production. Cell responses given by the characteristics of waves, are different, such as waveforms (sine or square), changes in biological effects and the type of cells that are exposed. In the past decade, there have been many studies on the effects of electromagnetic fields on the nervous system of humans and animals [2]-[4]. Extremely low frequency electromagnetic fields (ELFMF) exit in the right set of power lines close to residences, or the wiring of residential electricity or medical devices [5]. There are some reports on the various biological effects of ELFE, including interference with brain activity, behavioral and cognitive damage, alterations in locomotors activity, pain system, the N-methyl-D-aspartic acid receptor activity (NMDA) and messaging calcium in the hippocampus and the effect of heart rate and sleep at night [6]. Various Clinical studies related to the effects of electromagnetic waves on the nervous system have been carried out, showing the effects of radiation on brain activity, including decreased sensitivity to sensory stimuli, changes in bioelectrical activity of the brain and behavioral abnormalities [7].

Biochemical and physiological changes in the brain cause the release of neurotransmitters such as acetylcholine in the brain systemic circulation [1]. Since 1996, the World Health Organization has started International Project called electromagnetic fields Project, to gather evidence on the impact of electromagnetic radiation on people's health [8]. Moreover, low-frequency ultrasounds have been also used successfully to treat diabetic wounds in one study [9]. In recent years, numerous animal studies have been performed with low frequency electromagnetic field [10] [11].

Due to the fact that there are a lot of diseases associated with chronic pain, and dealing with them can be done with drugs that have many adverse effects to follow [5], the efficacy of alternative medicine of Extremely Low Frequency Magnetic Field (ELF-MF) on formalin-induced chronic pain in mice is reported [12].

\section{Materials and Methods}

\subsection{Animals}

Thirty two Male adult mice weighing $25 \pm 5$ g (Pasteur Institute, Karaj Production and Research Center, Iran) were used in this study. The animals were randomly divided into 4 groups of 8 in each. Animals housed under the following laboratory conditions: temperature $22^{\circ} \mathrm{C} \pm 1{ }^{\circ} \mathrm{C}$, humidity $40 \%-60 \%, 12: 12-\mathrm{L} / \mathrm{D}$ cycle, lights on at 07:00 h. Mice were maintained in polyethylene cages with food and water available ad libitum. Experiments were carried out between 10:00 and 14:00 h in the animal testing room. Mice were treated by the current regulations of Medical Science Research Center, Tehran Medical Branch, Islamic Azad University, Tehran, Iran, in accordance with the National Institutes of Health $(\mathrm{NIH})$ Guidelines for Care and Use of Laboratory Animals.

\subsection{Treatment \& Formalin Test}

First three groups of animals were exposed to the generator magnetic field intensity of 250 micro Tesla in 25,50 and $75 \mathrm{~Hz}$ frequencies, that checked by Teslameter, for half an hour daily for a week. One group considered as control and not exposed. Then, animals were injected about 15 - 20 micro liter Formalin (2.5\%) (SIGMA) subcutaneously to the sole of the right hind paws. Pain responses with the numbers $0,1,2,3$ recorded every 15 seconds for 60 minutes in the following guideline [13].

- Zero: animal walking on two legs perfectly balanced and weight is distributed on two legs.

- Score one: the animal's body weight does not tolerate on the injected foot and has trouble on walking.

- Score two: animal has a painful paw and kept it up and did not have contact with the floor.

- Score three: licking the painful paw or shaking it violently.

\subsection{Nora Pulsed Magnetic Field System Version NI931a}

The instrument (Figure 1) has been made for the research lab of Herbal Pharmacology Research Center that the 


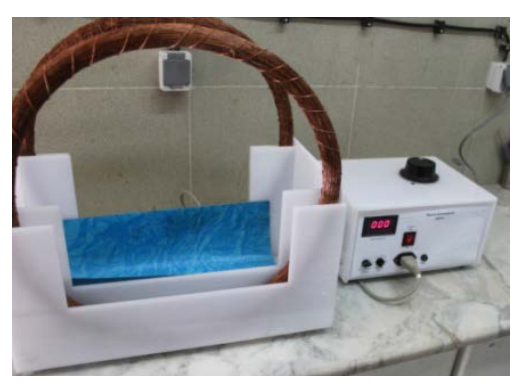

Figure 1. A view of the electromagnetic devices (small animal cage place: on the blue section).

project has been performed. It is constituted of flow generating device and Helmholtz coils. The system generated pulse waveform and applied it to the Helmholtz coils. In the space between the two coils a uniform magnetic field along the axis of the coils will create. More characteristics of the device is as follows: Changes in pulse frequency available in the range of 10 to $200 \mathrm{~Hz}$, Changes in pulse width available in the range of 1 to 2.5 milliseconds and Changes in pulse amplitude available in the range of zero to 300 volts.

\subsection{Statistical Analysis}

One-way ANOVA followed by Kruskal-Wallis analysis between groups exposed to a magnetic field, 25, 50 and 75 and control, were applied using ORIGIN 6 statistical software. All data were expressed as the mean \pm standard error of the mean (SEM).

\section{Results}

In the first (acute) phase of the formalin test (the first $10 \mathrm{~min}$ ) (Figure 2), the electromagnetic fields caused a significant reduction in pain symptoms compared to the control group. The pain reduction was most in $50 \mathrm{~Hz}$ magnetic field.

In the second (chronic) phase of the formalin test (within 16 to 60 minutes), magnetic field decreased pain scores induced by formalin significantly compared to the control group; the minimum effects induced by the frequency of $75 \mathrm{~Hz}$ and maximum analgesic efficacy observed at the $50 \mathrm{~Hz}$. The mean scores during the chronic phase are shown in Figure 3.

\section{Discussion and Conclusion}

The findings of this study showed that extremely low frequency electromagnetic fields leads to a significant increase in acute and chronic phases of pain threshold induced by formalin. In other words and in subsequent interpretation of these results chronic pain in mice was reduced.

The findings of this study correlates with other original basic research on analgesic effect of different frequencies of electromagnetic fields that were performed on rats and snails. Some clinicians tried low frequency electromagnetic fields effects on some arthritic pain [7]. In the most recent review synergistic effects of nitric oxide electromagnetic fields at very low frequencies have been reported [11]. Researchers use Electro-Magnetic treatment in patients with type 2 diabetes and in this way realized the increase of the effects of nitric oxide in comparison with the control group. This change primarily causes vasodilatation and increase blood flow in organs and secondly reduces the possible pain [14].

Thomas et al. (1977) presented that in all studies many factors have been caused vast variety in reported results including: animal species, the exposure duration, intensity, time-tested, frequency of the fields and other factors. In general, low frequency electromagnetic fields can reduce pain and anxiety in living organisms [15]. Baharara et al. (2008) examined the effect of electromagnetic fields on pain relief in chronic inflammatory pain model in rats and have shown that serotonin receptor antagonist and alpha 2 adrenergenic receptor antagonists inhibited significantly the effect of relieving pain [16]. Kitaoka and colleagues in Japan examined the impact of such low-frequency fields on increasing the secretion of corticosteroid hormones [12].

With regard to the above scientific assessment, low frequency electric and magnetic fields are important and must be considered as complementary therapy in medicine. 


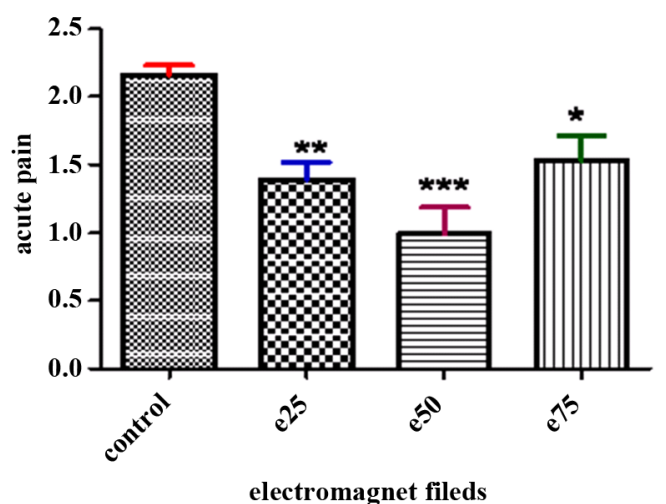

Figure 2. Mean of first (acute) phase of the formalin test (the first 10 minutes), in four groups; control, and 25,50 and $75 \mathrm{~Hz}$ (intense $250 \mu \mathrm{T}$ ) of electromagnet field for one week. ${ }^{*} \mathrm{p}<0.05,{ }^{* *} \mathrm{p}<0.01,{ }^{* * *} \mathrm{p}<0.001$.

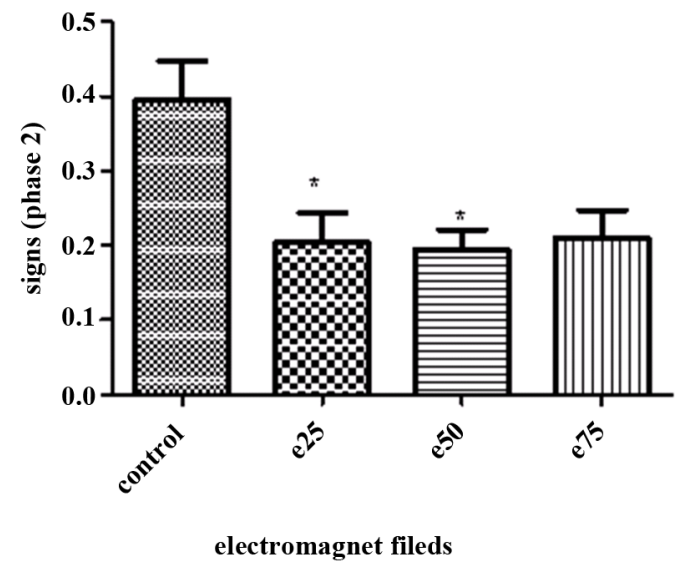

Figure 3. Mean of second (chronic) phase of the formalin test (the last 45 minutes), in four groups; control, and 25,50 and $75 \mathrm{~Hz}$ (intense 250 $\mu \mathrm{T})$ of electromagnet field for one week. ${ }^{*} \mathrm{p}<0.05,{ }^{* *} \mathrm{p}<0.01,{ }^{* * *} \mathrm{p}<$ 0.001 .

\section{Conclusion}

In general, it is concluded that low-frequency electromagnetic fields can affect the pain threshold in chronic pain in mice. The mechanisms involved and characteristics of its effectiveness on nervous system require further investigation.

\section{Acknowledgements}

The research was supported by the grant (No. 348) from the research deputy of Islamic Azad University, Tehran Medical Sciences Branch, Tehran, Iran. Authors would like to thank Dr. Azadeh Sonei for her valuable help in preparation of the manuscript.

\section{References}

[1] Andrea, A., Maria, G.C., Giorgio, B. and Bruno, B. (1997) Effects of Time-Variant Extremely-Low-Frequency (ELF) Electromagnetic Fields (EMF) on Cholinesterase Activity in Cells. European Journal of Prostiology, 157, 355-356.

[2] Nafisi, S., Atari, S., Kazami, R. and Hosseini, E. (2009) Effect of Low and Moderate Frequency Electromagnetic Fields on Stress Behavior in Rat. Journal of Ardebil Medical University, 5, 347-352.

[3] Akhtary, Z., Rashidy-Pour, A., Vafaei, A. and Jadidi, M. (2011) Effects of Extremely Low-Frequency Electromagnetic Fields on Learning and Memory and Anxiety-Like Behaviors in Rats. Journal of Semnan Medical Sciences University, 
12, 435-446.

[4] Miladi-Gorgi, H. and Vafaei, A. (2005) Effect of Systemic Injection of Vasopressin on Anxiety in Plus Maze in Mice. Journal of Guilan Medical Sciences, 14, 14-15.

[5] Jafari, H., Jahani-Hashemi, H. and Safari, A. (2006) Effects of Low Frequency Electromagnetic Fields on Withdrawal Syndrome Signs in Rat. Iranian Journal of Neurology, 14, 33-40.

[6] Rahmani, T., Bahrpeyma, F., Iranparvar, M. and Taghikhani, M. (2009) Study on Effect of Electromagnetic Therapy on Nitric Oxide Level and Ankle Brachial Index of Type 2 Diabetic Patients. Journal of Ardabil University of Medical Sciences, 14, 292-300.

[7] Martin, L.J., Koren, S.A. and Persinger, M.A. (2004) Thermal Analgesic Effect from Week, Complex Magnetic Fields and Pharmacological Interaction. Journal of Pharmacology Biochemistry and Behavior, 78, 217-227.

[8] Fiorani, M., Cantoni, O., Sestili, P., Conti, R., Nicolini, P., Vetrano, F. and Dachà, M. (1992) Electric and/or Magnetic Field Effects on DNA Structure and Function in Cultured Human Cells. Mutation Research Letters, 282, 25-29. http://dx.doi.org/10.1016/0165-7992(92)90069-t

[9] Amini, S. (2011) Effect of Low Ultrasound Waves on Diabetic Wounds Associated with Osteomyelitis in Clinical Trial. Iranian Journal of Diabetes and Metabolism, 10, 1390-1398.

[10] Baharara, J., Parivar, K., Ashraf, A. and Rostami, R. (2010) The Synergetic Effects of Low Frequency Electromagnetic Fields and Vitamin A on the Development of Skin in Balb/C Mice. Journal of Arak University of Medical Sciences, 12, $10-18$.

[11] Gharamaleki, H., Soleymani, R.J., Roshangar, L., Vatankhah, A. and Valipour, A. (2014) Effect of Extremely Low-Frequency Electromagnetic Field Exposure on Biomarkers of Oxidative Stress in Pregnant Rats. Journal of Iranian Association of Clinical Laboratory Doctors, 6, 31-35.

[12] Kitaoka, K., Kitamura, M., Aoi, S., Shimizu, N. and Yoshizaki, K. (2013) Chronic Exposure to an Extremely LowFrequency Magnetic Field Induces Depression-Like Behavior and Corticosterone Secretion without Enhancement of the Hypothalamic-Pituitary-Adrenal Axis in Mice. Journal of Bioelectromagnetics, 34, 43-51.

[13] Dennis, S. and Dobuisson, D. (1977) The Formalin Test: A Quantitative Study of the Effects of Analgesic Effects of Morphine. Neuroreport, 8, 10-14.

[14] Salunke, B.P., Umathe, S.N. and Chavan, J.G. (2014) Unvolvement of NMDA Receptor in Low-Frequency Magnetic Field-Induced Anxiety in Mice. Journal of Electromagnetic Biology and Medicine, 33, 312-326.

[15] Thomas, A.W., Kavaliers, M., Prato, F.S. and Assenkopp, K.P. (1977) Antinoceciptive Effects of Pulsed Magnetic Field in the Land Snail, Cepaeanemoralis. Neuroscience Letters, 222, 107-110.

[16] Baharara, J., Hadad, F., Ashraf, A. and Khanderoo, E. (2008) The Effect of Extremely Low Frequency Electromagnetic Field $(50 \mathrm{~Hz})$ on Induction of Chromosomal Damages on Bone Marrow Erythrocytes of Male Balb/C Mouse. Journal of Arak University of Medical Sciences, 12, 19-26. 